\title{
Analytic morphomics identifies predictors of new-onset diabetes after liver transplantation
}

\author{
Vaughn VM, Cron DC, Terjimanian MN, Gala ZS, Wang SC, Su \\ GL, Volk ML. Analytic morphomics identifies predictors of new- \\ onset diabetes after liver transplantation.
}

Abstract: Among liver transplant recipients, development of posttransplant complications such as new-onset diabetes after transplantation (NODAT) is common and highly morbid. Current methods of predicting patient risk are inaccurate in the pre-transplant period, making implementation of targeted therapies difficult. We sought to determine whether analytic morphomics (using computed tomography scans) could be used to predict the incidence of NODAT. We analyzed peri-transplant scans from 216 patients with varying indications for liver transplantation, among whom $61(28 \%)$ developed NODAT. Combinations of visceral fat, subcutaneous fat, and psoas area were considered in addition to traditional risk factors. On multivariate analysis adjusting for usual risk factors such as type of immunosuppression, subcutaneous fat thickness remained significantly associated with NODAT $(\mathrm{OR}=1.43,95 \% \mathrm{CI}$ $1.00-1.88, \mathrm{p}=0.047)$. Subgroup analysis showed that patients with lateronset of NODAT had higher visceral fat, whereas subcutaneous fat thickness was more correlated with earlier-onset of NODAT (using 10 months post-transplant as the cut-off).

Conclusion: Analytic morphomics may be used to help assess NODAT risk in patients undergoing liver transplantation.

\section{Valerie M. Vaughn ${ }^{\mathrm{a}, \boldsymbol{*}}$, David C. Cron $^{\mathrm{b}, \mathrm{c}, \star}$, Michael N. Terjimanian ${ }^{\mathrm{b}, \mathrm{c}}$, Zachary S. Gala ${ }^{\mathrm{b}, \mathrm{c}}$, Stewart C. Wang ${ }^{b, c}$, Grace L. Su ${ }^{b, d, e}$ and Michael L. Volk}

aDepartment of Internal Medicine, University of Michigan Medical School, ${ }^{b}$ Morphomic Analysis Group, University of Michigan Medical School, 'Department of Surgery, University of Michigan Medical School, ${ }^{\mathrm{V}} \mathrm{VA}$ Ann Arbor Health Care System and ${ }^{e}$ Division of Gastroenterology, University of Michigan Medical School, Ann Arbor, MI, USA

\begin{abstract}
Key words: analytic morphomics - body composition - cirrhosis - liver disease metabolic syndrome - new-onset diabetes after transplantation
\end{abstract}

\author{
Corresponding author: Valerie M. Vaughn, MD, \\ Department of Internal Medicine, University of \\ Michigan Medical School, 3110 Taubman Ctr, \\ SPC 5368, Ann Arbor, Ml 48109-5362, USA. \\ Tel.: +1 734936 4340; \\ fax: +1734936 3654; \\ e-mail:valmv@med.umich.edu \\ *These authors contributed equally to this \\ work. \\ Conflict of interest: None. \\ Accepted for publication 2 March 2015
}

Liver transplantation is the only cure for endstage liver disease and is an increasingly utilized treatment for hepatocellular carcinoma (HCC) in select populations. Despite improved survival with transplantation, recipients are at risk for a host of metabolic phenomena post-transplant due to transplant medications, resumption of previous dietary habits, and their underlying disease processes. New-onset diabetes after transplantation (NODAT) is particularly common, with a reported incidence of $10.8-61 \%$ (1-4), and is linked to increased cardiovascular events, mortality, and other complications (2, 5, $6)$.
Accurately predicting which patients will develop NODAT could lead to interventions to ameliorate these complications. Established risk factors for NODAT include prolonged prednisone use, tacrolimus immunosuppression, HCC, and hepatitis $\mathrm{C}(\mathrm{HCV})(1,7)$. It is known that body composition - specifically body mass index (BMI), waist circumference, and waist to hip ratio (i.e., "pear-shape") - is correlated with the development of diabetes in non-cirrhotic populations. However, these measurements are difficult to apply in pretransplant patients who have varying degrees of ascites, subcutaneous edema, and sarcopenia that make these measurements inaccurate. 
The emerging field of analytic morphomics has enabled us to make more precise measurements of body composition using computed tomography (CT) scans. It has been shown that morphomic measures of fat distribution and quantification of trunk musculature using CT scans can help quantify surgical risk in a variety of populations, including liver transplant recipients (8-12). In addition, it has been shown that visceral adiposity and relative skeletal muscle mass in non-transplant patients are associated with the development of insulin resistance $(13,14)$. To our knowledge, there have been no studies investigating the utility of these measures in predicting NODAT in liver transplant recipients.

We therefore aimed to determine whether analytic morphomics could be used to predict the incidence of NODAT in patients undergoing liver transplantation. We hypothesized that higher fat and lower trunk muscle size would be associated with NODAT.

\section{Methods}

\section{Study population}

This study was approved by the University of Michigan Institutional Review Board. This was a retrospective study of adult patients who underwent liver transplantation at a single large academic hospital between 2000 and 2011 with available peri-operative CT imaging. Eligible scans were those within $90 \mathrm{~d}$ of the operation that included the L4 vertebral level. We further narrowed this group to include only those patients without documented diabetes at the time of transplantation. Lastly, we excluded patients who died within the first 10 months post-transplant. This subset served as our study cohort (Fig. 1). The following demographic and clinical characteristics were collected retrospectively from the electronic medical records: recipient age, gender, race, height, weight, BMI, serum albumin, model for end-stage liver disease score, liver disease etiology (or etiologies if multiple; see Table S1 for descriptions), tobacco use, diabetes family history, hyperlipidemia diagnosis, and immunosuppressive regimen active at 10 months post-transplant (tacrolimus, cyclosporine, and prednisone). Chart review was performed using the electronic medical record search engine (EMERSE) to query clinical documentation of these characteristics (15). At conclusion of this step, $10 \%$ of charts were manually reviewed by a single physician reviewer for quality assurance.

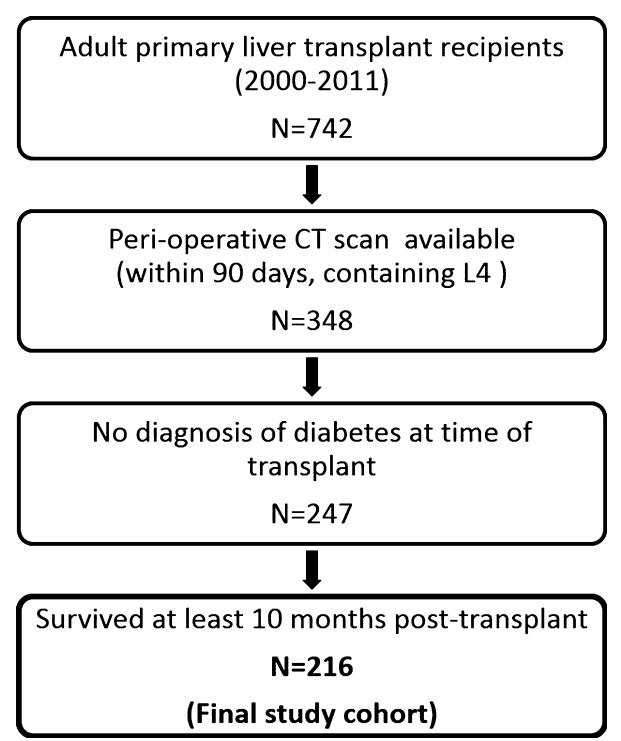

Fig. 1. Selection criteria. Patients were included if they had undergone transplant between 2000 and 2011 and had available peri-operative computed tomography (CT) imaging and did not carry a diagnosis of diabetes at the time of transplant. Patients who died within the first 10 months after transplant were excluded.

\section{Analytic morphomics}

Fig. 2 shows the morphomic measures considered in this analysis: total psoas area, visceral fat area, subcutaneous fat area, and anterior subcutaneous fat thickness. We also considered the following ratios: visceral fat to subcutaneous fat areas, visceral fat to total psoas areas, and subcutaneous fat to total psoas areas. CT scans were processed using semi-automated methods programmed into MAT-

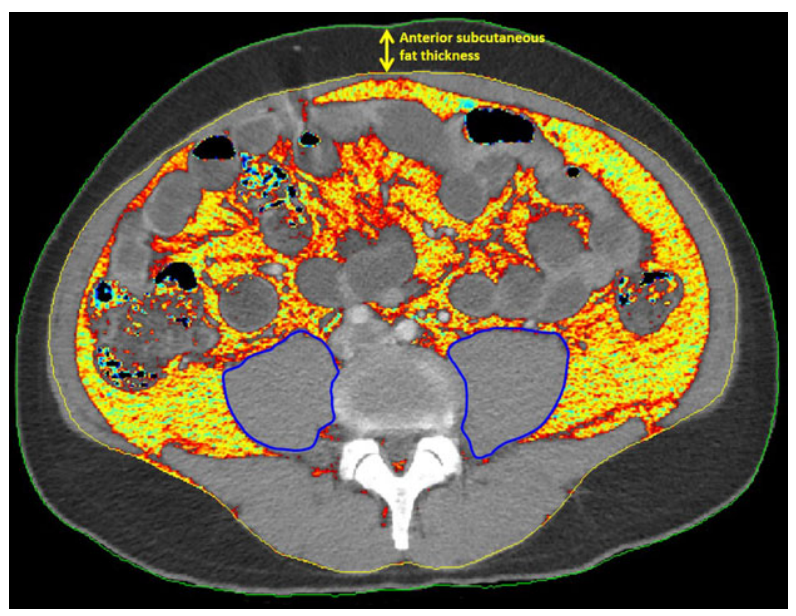

Fig. 2. Morphomic measurements. Morphomic measurements calculated from computed tomography scans are shown above. The fascia is outlined, and within this region, the visceral fat is portrayed by a heat map of density. The skin and the psoas muscles are outlined as well. 
LAB v. 2013a (MathWorks, Natick, MA, USA) after de-identified DICOM files were loaded in the morphomics server. The methods for measuring total psoas area are well described elsewhere $(8,9)$. Initially, each scan was anatomically indexed in a semi-automated fashion so that precise measurements could be made referenced to vertebral level (16). The cross-sectional areas of the left and right psoas muscles were computed inferior to the L4 vertebral level. These areas were summed to compute total psoas area, and this measurement was adjusted for the average Hounsfield units (density) of the psoas muscle to account for fatty infiltration (9). The resulting measure is termed lean psoas area.

Fat areas were measured at L4 as well for consistency. The fascial and skin envelopes were identified automatically after user-defined identification of several pelvic points and the linea alba. This was followed by user verification and editing as needed. Within the fascial envelope, the Hounsfield units of each pixel were calculated, and any pixels that fell within the density range of fat were deemed visceral fat. Between the skin and fascia contours, Hounsfield units were again used to identify subcutaneous fat. Because many larger patients have lateral regions of their subcutaneous fat cropped out of the CT images, we also included anterior subcutaneous fat thickness as a measure, which was available on all patients. This measure was computed as the shortest distance between the fascia and skin contours at the linea alba. Because a linear distance measure is more subject to random variation than area measurements, this measure was averaged across the T12 to L4 vertebral levels (17).

\section{Outcome}

The primary outcome of interest was combined NODAT - defined as incidence of NODAT at or beyond 10 months post-transplant. Because our follow-up began at 10 months post-transplant, we censored out patients who died prior to this 10 -month mark. Ten months has been used as the threshold in prior studies, as by this time, most patients are clinically stable, have been taken off steroids, and are therefore more likely to have stable blood sugars. This allows us to avoid misclassifying transient diabetes as NODAT (1). Other studies have used six or 12 months as thresholds; however, differences in incidence between the two periods were similar, and therefore, the exact time point is likely not significant $(4,6)$. For descriptive purposes, we also subdivided NODAT into "earlier-onset"
(NODAT incident prior to and persistent through the 10-month time point) and "lateronset" (NODAT incident beyond 10 months). We used EMERSE to retrospectively review the electronic medical records for mention of the following terms: NODAT, diabetes, DM, hyperglycemia, and insulin. For patients identified in this manner, we verified true diagnosis of NODAT according to the following American Diabetes Association criteria: $\mathrm{A} 1 \mathrm{C} \geq 6.5$, fasting plasma glucose $\geq 126$, oral glucose tolerance test at two $h \geq 200$, random glucose $\geq 200$, or any insulin use.

\section{Statistical analysis}

Descriptive statistics were computed for the study cohort. Categorical variables were summarized by frequency tables and compared using a chi-square test or Fisher's exact test, as appropriate. Continuous variables were summarized by mean and standard deviation and compared using a Student's $t$-test or a Wilcoxon rank-sum test, as appropriate. For regression analysis, morphomic variables were standardized by gender to facilitate interpretation of the regression coefficients, and the outcome was combined NODAT incidence (therefore including earlier-onset and later-onset). Logistic regression was used to identify factors associated with NODAT incidence on bivariate analysis. Variables with a $p$-value $\leq 0.20$ were considered "candidate predictors." Multivariate logistic regression was then applied to identify factors independently associated with NODAT incidence. All candidate predictors and clinically relevant variables were entered into the logistic regression analysis, and backward stepwise selection was used to determine a subset of adjustment covariates. Predictive ability of the models was assessed using the area under the receiver operating characteristic ( $C$-statistic). Model goodness of fit was verified using the Hosmer-Lemeshow test. All statistical analysis was performed using STATA v. 13 (College Station, TX, USA).

\section{Results}

Two hundred and sixteen patients met our inclusion criteria. Table 1 displays the demographic and clinical characteristics of this cohort. The most common underlying reasons for liver transplantation were $\mathrm{HCV}(37.5 \%)$, alcoholic cirrhosis $(26.9 \%), \operatorname{HCC}(26.4 \%)$, and cholestatic disease $(19.9 \%)$. The majority of patients $(79.6 \%)$ were on tacrolimus, and $42.1 \%$ of patients were on prednisone at 10 months post-transplant. The overall 
Table 1. Patient characteristics by NODAT status

\begin{tabular}{|c|c|c|c|c|}
\hline & Overall & No NODAT & Combined NODAT & $p$-Value \\
\hline Total n & 216 & 155 & 61 & \\
\hline Age & $49.5 \pm 10.1$ & $49.5 \pm 10.3$ & $49.0 \pm 9.9$ & 0.63 \\
\hline Male gender & $60.6 \%(131)$ & $59.4 \%(92)$ & $63.9 \%(39)$ & 0.54 \\
\hline Non-white race & $17.1 \%(37)$ & $15.5 \%(24)$ & $21.3 \%(13)$ & 0.31 \\
\hline BMI & $27.0 \pm 5.7$ & $26.7 \pm 5.8$ & $27.7 \pm 5.6$ & 0.24 \\
\hline MELD & $18.6 \pm 7.6$ & $18.7 \pm 7.6$ & $18.2 \pm 7.5$ & 0.68 \\
\hline Albumin & $2.8 \pm 0.6$ & $2.8 \pm 0.6$ & $2.8 \pm 0.6$ & 0.46 \\
\hline Tobacco use (past year) & $24.1 \%(52)$ & $20.0 \%(31)$ & $34.4 \%(21)$ & 0.03 \\
\hline Hyperlipidemia & $13.0 \%(28)$ & $15.5 \%(24)$ & $6.6 \%(4)$ & 0.09 \\
\hline Cardiovascular disease & $2.3 \%(5)$ & $3.2 \%(5)$ & 0 & 0.33 \\
\hline Diabetes family history & $39.8 \%(86)$ & $37.4 \%(58)$ & $45.9 \%(28)$ & 0.25 \\
\hline Tacrolimus & $79.6 \%(172)$ & $78.1 \%(121)$ & $83.6 \%(51)$ & 0.36 \\
\hline Cyclosporine & $20.4 \%(44)$ & $21.9(34)$ & $16.4(10)$ & 0.36 \\
\hline Prednisone & $42.1 \%(91)$ & $40.6 \%(63)$ & $45.9 \%(28)$ & 0.48 \\
\hline \multicolumn{5}{|l|}{ Liver pathology } \\
\hline Alcoholic & $26.9 \%$ (58) & $27.1 \%(42)$ & $26.2 \%(16)$ & 0.90 \\
\hline Autoimmune hepatitis & $4.6 \%(10)$ & $3.9 \%(6)$ & $6.6 \%(4)$ & 0.47 \\
\hline Hepatocellular carcinoma & $26.4 \%(57)$ & $26.5 \%(41)$ & $32.8 \%(20)$ & 0.35 \\
\hline Cholestatic $^{\star}$ & $19.9 \%(43)$ & $25.2 \%(39)$ & $6.6 \%(4)$ & 0.004 \\
\hline Fulminant & $3.7 \%(8)$ & $3.9 \%(6)$ & $3.3 \%(2)$ & 0.84 \\
\hline Hepatitis B virus & $6.0 \%(13)$ & $6.5 \%(10)$ & $4.9 \%(3)$ & 0.67 \\
\hline Hepatitis C virus* & $37.5 \%(81)$ & $31.6 \%(49)$ & $52.5 \%(32)$ & 0.005 \\
\hline NAFLD & $6.5 \%(14)$ & $7.1 \%(11)$ & $4.9 \%(3)$ & 0.56 \\
\hline Other & $8.8 \%(19)$ & $7.1 \%(11)$ & $13.1 \%(8)$ & 0.17 \\
\hline
\end{tabular}

Combined NODAT includes both earlier-and later-onset NODAT.

NODAT, new-onset diabetes after transplantation.

${ }^{*}$ Represents a significant difference when comparing no NODAT to combined NODAT $(p \leq 0.05)$.

incidence of NODAT was $28.2 \%$ (61 patients). Of these patients, $77.1 \%$ (47 patients) had earlieronset NODAT (present at 10 months post-transplant) and $23.0 \%$ (14 patients) had later-onset NODAT (incident beyond 10 months post-transplant). Of this latter group, the median time from transplant to diagnosis of NODAT was 26 months.

Table 2 displays the estimated (unadjusted) parameters for morphomic variables from the logistic regression analyses of the primary outcome (combined NODAT). In bivariate analysis, anterior subcutaneous fat thickness was the only significant morphomic predictor of NODAT (odds ratio $[\mathrm{OR}]=1.43$ per standard deviation increase, $95 \%$ CI $1.07-1.92, \mathrm{p}=0.02$ ). Other variables significantly associated with NODAT incidence in bivariate analysis included tobacco use in the past year $(p=0.03)$, HCV $(p=0.005)$, and cholestatic disease (which was protective; $\mathrm{p}=0.004$ ). The overall distribution for anterior subcutaneous fat thickness was skewed right, and values ranged from 2.3 to $50.9 \mathrm{~mm}$. There was a strong positive correlation between anterior subcutaneous fat thickness and subcutaneous fat area $(r=0.80)$.

In multivariate analysis, subcutaneous fat thickness remained significantly associated with
Table 2. Estimated morphomic coefficients from bivariate logistic regression for combined NODAT

\begin{tabular}{|c|c|c|}
\hline & OR & $\mathrm{p}$-Value \\
\hline \multicolumn{3}{|l|}{ Morphomic features } \\
\hline Subcutaneous fat area (SD units) & 1.18 & 0.262 \\
\hline Visceral fat area (SD units) & 1.07 & 0.663 \\
\hline Visceral fat to subcutaneous fat (ratio) & 1.07 & 0.652 \\
\hline Anterior subcutaneous fat thickness (SD units)* & 1.43 & 0.017 \\
\hline Lean psoas area (SD units) & 1.10 & 0.513 \\
\hline Subcutaneous fat to lean psoas (ratio) & 1.01 & 0.469 \\
\hline Visceral fat to lean psoas (ratio) & 1.02 & 0.622 \\
\hline
\end{tabular}

Combined NODAT includes both earlier- and later-onset NODAT. NODAT, new-onset diabetes after transplantation.

${ }^{*}$ Represents a significant difference when comparing no NODAT to combine NODAT $(p=0.05)$.

$\operatorname{NODAT}(\mathrm{OR}=1.43,95 \%$ CI $1.00-1.88, \mathrm{p}=0.047)$, along with HCV, and "other" diagnosis (see Table S1 for description of "other" diagnosis). BMI was not significantly different. Cholestatic disease was removed from multivariate analysis because it was mutually exclusive with $\mathrm{HCV}$ and therefore predicted each other in modeling. The Cstatistic for this model was 0.70 . The covariates in this model were then used to calculate adjusted rates of NODAT incidence. Fig. 3 shows these 
Adjusted Incidence of Combined NODAT by Tertile of Subcutaneous Fat Thickness.

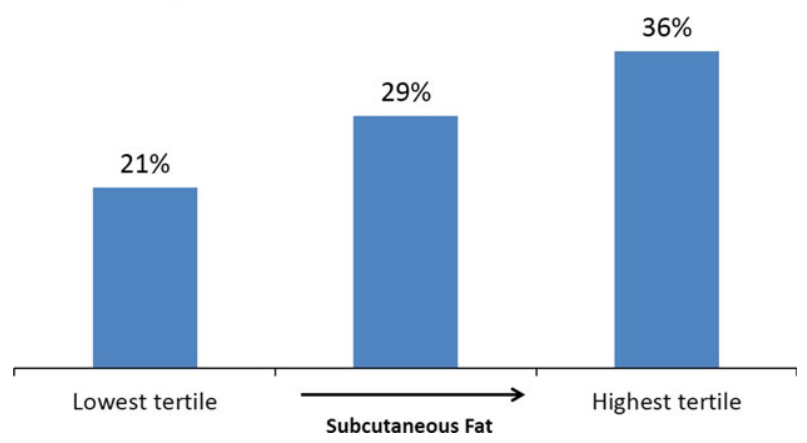

Fig. 3. Adjusted incidence of combined new-onset diabetes after transplantation (NODAT) by tertile of subcutaneous fat thickness. After adjustment for hepatitis C, hyperlipidemia, and "other" diagnoses, patients with the most anterior subcutaneous fat had a 1.7-fold higher incidence of NODAT compared to the patients with the least anterior subcutaneous fat (36\% compared with $21 \%$ ). Combined NODAT includes both earlier- and later-onset NODAT.

rates by gender-stratified tertile of anterior subcutaneous fat thickness. Patients with the most anterior subcutaneous fat had 1.7-fold higher incidence of NODAT compared to patients with the least anterior subcutaneous fat.

For our secondary analysis, we divided patients into earlier-onset and later-onset NODAT. Table 3 shows the median morphomic values for the following three groups of patients: those without NODAT, those with earlier-onset NODAT, and those with later-onset NODAT. Compared to patients without NODAT, visceral fat area and the ratio of visceral fat to lean psoas area were significantly larger in the later-onset NODAT group (visceral fat area: $\mathrm{p}=0.02$; ratio: $\mathrm{p}=0.01$ ). Compared to patients without NODAT, anterior subcutaneous fat thickness was significantly larger in both the earlier-onset group $(p=0.04)$ and the later-onset group $(\mathrm{p}=0.02)$.

As a sensitivity analysis, we compared the morphomic characteristics of patients with diabetes at the time of transplant (excluded initially from our main cohort) and patients in our cohort who developed NODAT (Table S2). Compared to diabetic liver transplant candidates, the onset NODAT patients had significantly lower visceral fat $(p=0.002)$, larger lean psoas area $(p=0.02)$, and smaller ratios of subcutaneous fat to leas psoas area $(p=0.001)$ and smaller visceral fat to lean psoas area $(p<0.001)$. The later-onset NODAT patients were similar to the diabetic comparison group in all morphomic aspects. The patients in our study cohort who did not develop NODAT were significantly different from the diabetic comparison group in all morphomic aspects (lower fat and larger muscles in the patients without NODAT) except the ratio of visceral to subcutaneous fat.

Tacrolimus use was not found to be significant in bivariate or multivariate modeling. As a sensitivity analysis, we ran the models again using average tacrolimus trough levels from date of transplant to six months post-transplant (data available for all but six patients). By itself, average tacrolimus trough was a significant predictor of NODAT, but this measure was not significant when adjusting for subcutaneous fat thickness or when included in the multivariate model.

\section{Discussion}

This is the first study evaluating analytic morphomics in a liver transplant population to determine associations with NODAT. We found that subcutaneous fat thickness was independently associated with NODAT, even after adjustment for other clinical and demographic characteristics. In addition, visceral fat area and the ratio of visceral fat to lean psoas were associated with later-onset (beyond 10 months) NODAT. The incidence of NODAT in our study was $28.2 \%$ - well within typical values found in other studies (1-4). Similar to other studies, the variables we found to be associated with

Table 3. Median morphomic values by timing of NODAT incidence

\begin{tabular}{lccc}
\hline & No NODAT $(n=152)$ & Earlier-onset NODAT $(n=47)$ & Later-onset NODAT $(n=14)$ \\
\hline Visceral fat area $\left(\mathrm{mm}^{2}\right)$ & 6234 & 6975 & $10147^{*}$ \\
Subcutaneous fat area $\left(\mathrm{mm}^{2}\right)$ & 15474 & 15493 & 16240 \\
Visceral fat to subcutaneous fat (ratio) & 0.5 & 0.4 & 0.6 \\
Anterior subcutaneous fat thickness $(\mathrm{mm})$ & 15 & $18^{*}$ & $23^{*}$ \\
Lean psoas area $\left(\mathrm{mm}^{2}\right)$ & 1545 & 1843 & 1525 \\
Subcutaneous fat to lean psoas (ratio) & 9.3 & 10.2 & 11.5 \\
Visceral fat to lean psoas (ratio) & 3.8 & 3.7 & $6.9^{*}$ \\
\hline
\end{tabular}

NODAT, new-onset diabetes after transplantation; Earlier-onset, NODAT incident prior to and persistent through 10 months post-transplant; Later-onset, NODAT incident beyond 10 months.

*Represents a significant difference when compared to those without NODAT $(p \leq 0.05)$ 
NODAT included tobacco use and HCV, with cholestatic disease being protective from NODAT. However, not all previously proven risk factors (e.g., HCC and tacrolimus use) were found to be statistically significant, likely due to the small sample size and to the relatively large percentage of patients on tacrolimus rather than cyclosporine or other agents.

As with all retrospective studies, we were limited by the data collection available in the chart, and it is possible that not all patients had adequate testing for NODAT or that risk factors were not mentioned within the chart. We attempted to mitigate this factor by searching broadly for multiple search terms and including both chart review and laboratory data in determining NODAT status. In addition, this study is susceptible to selection bias, as only those patients who had CT scans and who maintained follow-up could be included. We were not able to measure all factors associated with NODAT as caloric intake and exercise are difficult to measure retrospectively. Although we attempted to correct for any measured confounders using logistic regression, retrospective studies are prone to these types of biases and do not prove causality. A larger prospective study would need to be undertaken to verify our findings. Concerning our definition of NODAT, there is no clear consensus on a temporal definition; we chose presence at 10 months post-transplant as our threshold. We also ran the analyses using survival analysis techniques (time until NODAT onset) and we observed the same findings, with subcutaneous fat thickness as the only significant morphomic predictor (data not shown). Finally, this is a single-center study, and therefore, results from our population may not be generalizable to other centers.

Other studies have shown visceral fat at the L4 umbilicus and visceral to subcutaneous fat ratio to be related to surgical risk and diabetes; however, we did not find these measurements to be significant in our cohort $(12,13)$. In our sample, we found that subcutaneous fat could not be adequately measured in $12 \%$ of patients due to cropping of CT scan images on the lateral aspects of the field, presumably underestimating subcutaneous fat for these patients. By using midline subcutaneous fat, which is correlated with total subcutaneous fat, this limitation was eliminated leaving us with an easy-to-measure variable that is correlated with NODAT. Midline subcutaneous fat could also reflect a different body shape or fat distribution that predisposes to diabetes. Alternatively, fat thickness may be less confounded by body size, making it a more reliable measure of fat. We did observe significantly higher visceral fat in the later-onset NODAT patients and more similarities between the morphomics of lateronset patients and patients who had diabetes pretransplant. This could possibly point to a biochemical difference between earlier- and lateronset diabetes. Patients who develop later-onset NODAT may be genetically or physically pre-disposed to diabetes (i.e., they would have developed diabetes eventually without a transplant). Those with earlier-onset NODAT may instead have developed diabetes due to immunosuppression or other transplant-related factors. Due to small sample size, we were unable to consider timing of NODAT incidence in our primary multivariate analysis. This finding merits further study as this would help to reveal a population at risk for NODAT that might not be normally considered at risk.

Given the high morbidity associated with NODAT, it is imperative that high-risk patients are identified early so that modifiable risk factors can be addressed and corrected. The 2003 International Consensus Guidelines recommend evaluating and identifying patients at risk for NODAT during the initial consult for transplantation (18). Pre-transplant patients often undergo pre-operative crosssectional imaging in the course of their work-up and for HCC screening. By using these already available imaging scans, we can further identify atrisk patients who would merit individualized weight, diet, and exercise counseling, which has been shown to prevent diabetes in high-risk patients (19). Additional interventions could target immunosuppressive therapy by focusing on minimizing steroids (18). In conclusion, our study shows that analytic morphomics may be useful for assessing NODAT risk in patients undergoing liver transplantation.

\section{Financial support}

None.

\section{References}

1. Mirabella S, Brunati A, Ricchiuti A, Pierini A, FranChello A, Salizzoni M. New-onset diabetes after liver transplantation. Transplant Proc 2005: 37: 2636.

2. Laryea M, Watt KD, Molinari M et al. Metabolic syndrome in liver transplant recipients: prevalence and association with major vascular events. Liver Transpl 2007: 13: 1109.

3. Saab S, Shpaner A, Zhao Y et al. Prevalence and risk factors for diabetes mellitus in moderate term survivors of liver transplantation. Am J Transplant 2006: 6: 1890.

4. Stegall MD, Everson G, Schroter G, Bilir B, Karrer F, KAм I. Metabolic complications after liver transplantation. 


\section{Vaughn et al.}

Diabetes, hypercholesterolemia, hypertension, and obesity. Transplantation 1995: 60: 1057.

5. Malik S, Wong ND, Franklin SS et al. Impact of the metabolic syndrome on mortality from coronary heart disease, cardiovascular disease, and all causes in United States adults. Circulation 2004: 110: 1245.

6. Lunati ME, Grancini V, Agnelli F et al. Metabolic syndrome after liver transplantation: short-term prevalence and pre- and post-operative risk factors. Dig Liver Dis 2013: 45: 833.

7. Bodziak KA, Hricik DE. New-onset diabetes mellitus after solid organ transplantation. Transpl Int 2009: 22: 519.

8. Englesbe MJ, Patel SP, He K et al. Sarcopenia and mortality after liver transplantation. J Am Coll Surg 2010: 211: 271.

9. Englesbe MJ, Lee JS, He K et al. Analytic morphomics, core muscle size, and surgical outcomes. Ann Surg 2012: 256: 255.

10. Waits SA, Kim EK, Terjimanian MN et al. Morphometric age and mortality after liver transplant. JAMA Surg 2014: 149: 335 .

11. Englesbe MJ, Terjimanian MN, Lee JS et al. Morphometric age and surgical risk. J Am Coll Surg 2013: 216: 976.

12. Clark W, Siegel EM, Chen YA et al. Quantitative measures of visceral adiposity and body mass index in predicting rectal cancer outcomes after neoadjuvant chemoradiation. J Am Coll Surg 2013: 216: 1070.

13. Hayashi T, Boyko EJ, McNeely MJ, Leonetti DL, Kahn SE, Fuлmото WY. Visceral adiposity, not abdominal subcutaneous fat area, is associated with an increase in future insulin resistance in Japanese Americans. Diabetes 2008: 57: 1269
14. PARK BS, YoOn JS. Relative skeletal muscle mass is associated with development of metabolic syndrome. Diabetes Metab J 2013: 37: 458.

15. Hanauer DA. EMERSE: The Electronic Medical Record Search Engine. AMIA Annu Symp Proc 2006: 941.

16. Zhang P, Parenteau C, Wang L et al. Prediction of thoracic injury severity in frontal impacts by selected anatomical morphomic variables through model-averaged logistic regression approach. Accid Anal Prev 2013: 60: 172

17. Lee JS, Terjimanian MN, Tishberg LM et al. Surgical site infection and analytic morphometric assessment of body composition in patients undergoing midline laparotomy. J Am Coll Surg 2011: 213: 236.

18. Davidson J, Wilkinson A, Dantal J et al. New-onset diabetes after transplantation: 2003 International consensus guidelines. Proceedings of an international expert panel meeting. Barcelona, Spain, 19 February 2003. Transplantation. 2003: 75 (10 Suppl): SS3.

19. Lindström J, Eriksson JG, Valle TT et al. Prevention of type 2 diabetes mellitus by changes in lifestyle among subjects with impaired glucose tolerance. N Engl J Med 2001: 344: 1343 .

\section{Supporting Information}

Additional Supporting Information may be found in the online version of this article:

Table S1. Liver disease diagnostic categories.

Table S2. Median morphomic values by pre-transplant diabetes and NODAT status. 